\title{
Condiciones de trabajo y salud de una universidad venezolana
}

\author{
Maritza Rojas-Martini, MSC, ${ }^{(1)}$ Guido Squillante, M en SO, (1) \\ Carlos Espinoza, Ing en $\mathrm{S}^{\left({ }^{(1)}\right.}$
}

Rojas-Martini M, Squillante G, Espinoza C. Condiciones de trabajo y salud de una universidad venezolana. Salud Publica Mex 2002;44:413-421. El texto completo en inglés de este artículo está disponible en: http://www.insp.mx/salud/index.html

\section{Resumen}

Objetivo. Identificar los procesos peligrosos a los que están expuestos los trabajadores de una universidad venezolana como consecuencia de sus actividades laborales. Material y métodos Se realizó un diagnóstico de las condiciones de exposición laboral de los trabajadores de una universidad venezolana (UN), de sus potenciales procesos peligrosos (PP) y de sus causas, mediante un estudio de corte transversal, efectuado en 1999. Se estudiaron 90 trabajadores de uno u otro sexo, pertenecientes a 24 áreas clasificadas como críticas por las autoridades de la UN. Se aplicaron una encuesta de área y una personal. En ésta, se investigaron car acterísticas demográficas, historia clínica ocupacio nal, procesos peligrosos ocupacionales, uso de equipos de protección personal (EPP), conocimiento de riesgos laborales y hábitos. Se practicaron exámenes clínico-toxicológicos, entre otros. Se identificaron los principales PP químicos, físicos, biológicos, mecánicos, ergonómicos y psicosociales y el grado de riesgo (GR) por cada área. Se aplicaron medidas de tendencia central y de dispersión, así como también medidas de asociación y significancia estadística, pertinentes a cada caso. Resultados Se obtuvo una correlación positiva, significativa entre la antigüedad en la UN y el número de signos y síntomas referidos por los trabajadores, pero no entre estos últimos y la edad o el sexo. Los síntomas en su mayoría resultaron no específicos. Conclusiones En la UN se presentan variados procesos potencialmente peligrosos en las diferentes áreas laborales debido a las características inherentes a su misión. Se ha-

\author{
Rojas-Martini M, Squillante G, Espinoza C. \\ Work and health conditions \\ at a Venezuelan university. \\ Salud Publica Mex 2002;44:413-421. \\ The English version of this paper \\ is available at: http://www.insp.mx/salud/index.html
}

\begin{abstract}
A bstract
Objective. To identify occupational exposure to hazards amongVenezuelan university (UN) workers. Material and MethodsA descriptive cross-sectional study was conducted in 1999 to assess occupational exposure among UN workers. Potentially hazardous events (PH) and their causes were identified. The study population consisted of 90 workers of both genders, working in any of the 24 areas classified as critical by university authorities. W ork area and individual surveys were carried out. Data collected included demographic characteristics, occupational clinical history, hazardous occupational exposures, use of personal protection equipment, and knowledge about occupational risks and habits. Clinical-toxicological tests were performed.The major chemical, physical, biolo gical, mechanical, and ergonomic PH were identified, as well as the degree of risk (DR) for each area. Central tendency and dispersion measures were calculated, in addition to association and statistical significance measures, as appropriate. Results. A positive, significant correlation was found between time working in the university and the number of signs and symptoms reported by workers. No correlation was fo und between the number of signs and symptoms and age and gender. Symptoms were mainly non-specific. Conclusions There are several potentially hazardous processes in the different occupational areas of the university. Recommendations are issued to implement prevention programs with appropriate recording of occupational accidents, and morbidity and mortality, to aid in the timely detection of occupational illnesses and
\end{abstract}

(1) Centro de Investigaciones Toxicológicas de la Universidad de Carabobo, Valencia, C arabobo, Venezuela.

Fecha de recibido: 23 de marzo de 2001 - Fecha de aprobado: 15 de marzo de 2002

Solicitud de sobretiros: Dra. Maritza Rojas-Martini. Instituto de Investigaciones Toxicológicas, Universidad de Carabobo. Calle 144 N Río-211 La C eiba, Valencia, Carabobo,Venezuela.

Correo electrónico: martini@ telcel.net.ve 
cen las recomendaciones pertinentes para instaurar programas que den prioridad a las acciones preventivas, que lleven registros adecuados sobre siniestralidad laboral, morbilidad y mortalidad, y que permitan la detección temprana de enfermedades y accidentes ocupacio nales. El texto completo en inglés de este artículo está disponible en:http:/ /www.insp.mx/salud/index.html

Palabras clave: salud ocupacional; univer sidades; instituciones académicas; Venezuela accidents. The English version of this paper is available at: http://www.insp.mx/salud/index.html

Key words: occupational health; universities; academic institutions; Venezuela
L os programas de seguridad y salud ocupacional (PSSO), que se ejecutan o deberían ejecutarse en instituciones académicas y de investigación, enfrentan muchos retos debido a la amplia variedad de procesos potencialmente peligrosos (PP) químicos, biológicos, físicos, ergonómicos o psicosociales, presentes en sus áreas laborales. Los países desarrollados cuentan, en su mayoría, con PSSO en las instituciones académicas. ${ }^{1-3}$ En los países en desarrollo, como Venezuela, estos programas o no existen, o no se ejecutan debidamente por la carencia de suficientes recursos presupuestarios, lo que ocasiona la imposibilidad de ofrecer a nuestros trabajadores servicios de prevención apropiados, y control de accidentes y de enfermedades ocupacionales.

No es extraño el hecho de que universidades venezolanas donde existen facultades de ciencias de la salud o de medicina, con programas de posgrado en salud ocupacional, posean unas condiciones laborales que distan mucho de ser las más adecuadas. En nuestro país, a pesar de que en 1986 se estableció una política nacional de salud ocupacional expresada en la Ley Orgánica de Prevención, Condiciones y Medio Ambiente de Trabajo (LOPCYMAT), ${ }^{4,5}$ no existe un sistema de registro confiable que facilite la determinación exacta de la ocurrencia de accidentes y enfermedades ocupacionales, aun cuando las investigaciones indiquen una frecuencia muy alta de accidentes de trabajo. ${ }^{6}$ Esta situación nacional es extrapolable al grupo específico de las instituciones universitarias.

La revisión de la literatura hecha por los autores de este trabajo evidencia la carencia de estudios de este tipo en nuestras instituciones académicas. Conscientes de la importancia que las condiciones de trabajo tienen sobre la salud y el bienestar de los trabajadores y alumnos, el Centro de Investigaciones Toxicológicas de la Universidad de Carabobo (CITUC), hizo un diagnóstico de las condiciones de exposición laboral de los trabajadores de una universidad venezolana (que llamaremos UN por razones de confidencialidad), de sus potenciales procesos peligrosos y de la identificación de las causas que originan una morbilidad, considerada por las autoridades de dicha universidad, como "alta". Este trabajo consistió en identificar los procesos peligrosos presentes en la UN, analizar estadísticamente e interpretar los resultados con el fin de definir prioridades de acción para el control de los mismos.

\section{Material y métodos}

El estudio corresponde a una investigación descriptiva, de corte transversal, de carácter exploratorio. La UN es gubernamental, con aproximadamente 6000 alumnos y 944 empleados, distribuidos en tres categorías: 484 docentes, 403 administrativos y 57 obreros. Tiene siete facultades: Ingeniería industrial, Mecánica, Agronomía, Electrónica, Informática, Producción animal y Arquitectura. El estudio se hizo en el último trimestre de 1999.

La población a estudiar fue previamente establecida por las autoridades de la UN, quienes elaboraron un listado de 120 trabajadores (personal docente, administrativo y obrero), distribuidos en 26 áreas. De éstas, se estudiaron 24 (92.3\%). Se incluyó sólo al personal que se encontraba presente al momento de la aplicación de la encuesta. La muestra final estuvo constituida por $90(75 \%)$ trabajadores -59 (65.5\%) del sexo masculino y $31(34.5 \%)$, del femenino-. Los 30 restantes (siete docentes, 13 administrativos y 10 obreros), estuvieron ausentes por permiso o por incapacidad médica, etcétera. El criterio utilizado por la UN para la selección de la población obedeció a varias razones, desde quejas de los trabajadores de sufrir patologías supuestamente relacionadas con la exposición laboral, hasta laborar en áreas consideradas como críticas por los representantes gremiales.

Se hizo inspección ocular a las áreas, incluyendo la aplicación de una encuesta. Esta consistió en un cuestionario para indagar características estructurales, tipo 
de iluminación, ventilación, temperatura, condiciones de seguridad, presencia de equipos de protección* personal (EPP), identificación de PP, etcétera. Esta encuesta ha sido validada en diversos estudios realizados por el CITUC a empresas que contratan sus servicios y es adaptada a cada estudio en particular. Sus resultados no han sido publicados por estrictas razones de confidencialidad.

Una vez identificados los PP en las áreas evaluadas se procedió a agruparlos según la clasificación tradicional establecida. ${ }^{7, *}$ Se seleccionaron los principales procesos químicos, mecánicos, físicos, psicosociales y ergonómicos, presentes en las áreas laborales.

Se aplicó una encuesta personal a cada trabajador, mediante la cual se obtuvo información demográfica, familiar, denominación del cargo, historia clínica ocupacional, de salud, PP ocupacionales, uso de EPP, estilos de vida (hábito alcohólico, tabáquico), ingesta de medicamentos, historia de exámenes clínicotoxicológicos, etcétera. Para obtener estos datos un médico ocupacional y un toxicólogo del CITUC aplicaron en forma personal un cuestionario a cada trabajador. La inspección ocular y las encuestas personales se tomaron en cuatro días consecutivos.

La selección de los ítems y preguntas para ambas encuestas se llevó a cabo de acuerdo con los antecedentes existentes en estudios similares y considerando el juicio y experiencia profesional de los investigadores en el área de evaluación de riesgos.

El establecimiento del grado de riesgo (GR), se fundamentó tanto en la "Nomenclatura y valoración utilizada en la evaluación de riesgos" de Select-España, ${ }^{\ddagger}$ como en el criterio profesional. Se utilizó una escala derivada de evaluar, por un lado, la intensidad del riesgo (IR) y por el otro, las características o patrones de exposición (CE), de los trabajadores estudiados. La IR se calificó por los resultados tanto de la inspección ocular como de la encuesta de área. Su valoración es: $1=$ leve, $2=$ moderado, $3=$ severo, $4=$ muy severo. Las CE de la población estudiada se calificaron con base en número de individuos expuestos por área, tipos y concentraciones de agentes contaminantes, factores de riesgo, duración de la exposición,

\footnotetext{
* Estrada J. 1999. Programa de Evaluación de Factores de Riesgo. Disponible en:http://www.cepis.org.pe/eswww/ocupfile//airo.doc [C onsultado: 23/09/2000].

₹ Select. Recursos Empresariales. N omenclatura y valoración utilizada en la evaluación de riesgos. Disponible en: http://www.selectett.es/prevenir/dermanu.htm [C onsultado: 10/04/2000].
}

signos y síntomas referidos, que pudiesen asociarse con los agentes contaminantes ocupacionales. Su valoración es como sigue: $1=$ baja, $2=$ mediana, $3=a l t a$, 4=muy alta. El diagnóstico final del GR, para cada área se derivó de los dos parámetros mencionados, y su valoración es: $1=$ bajo, $2=$ moderado, $3=$ =alto, $4=$ muy alto.

Los datos se resumieron en cuadros apropiados, utilizando para ello frecuencias absolutas y relativas. Se aplicaron medidas de tendencia central y de dispersión, y medidas de asociación y significancia estadística, pertinentes a cada caso.

\section{Resultados}

El promedio de edad de los empleados estudiados fue de 40.4 años, con un rango de 18 a 59 años y una desviación estándar $(D E)$ de 9.05. De éstos, $13(14.4 \%)$ pertenecían al personal docente, $60(66.7 \%)$ al administrativo y $17(18.9 \%)$, al obrero. El promedio de antigüedad fue de 4.7 años, rango 0.17-25 y DE 8.19.

Las áreas fueron debidamente codificadas y la distribución del personal estudiado se observa en el cuadro I.

Se determinó que en el área con el personal más numeroso (biblioteca), 11 personas (38\%) tienen una antigüedad en la UN en el rango de 20 a 25 años. Esta área fue seguida de computación, donde el mayor número $(6 ; 40 \%)$ estaba entre 15 y 19 años y mantenimiento en donde el mayor número $(5 ; 36 \%)$ se encontró en el rango de 0 a 4 años de antigüedad (cuadro I).

En el cuadro II se presenta el diagnóstico final del grado de riesgo para cada área.

Las dependencias con GR 3 constituyen 31.1\% de la población estudiada. Con relación al conocimiento de los diferentes tipos de riesgo laborales, por parte de los trabajadores, las respuestas afirman que $78(86.6 \%)$ de los trabajadores conocen los riesgos a que están sometidos en su trabajo y sus consecuencias.

Con relación a los PP de tipo químico, el más frecuente fue el polvo (65.6\%), seguido de solventes orgánicos $(25.6 \%)$ y ácidos fuertes (22.2\%). En los de tipo mecánico, las maquinarias ocuparon el primer lugar $(17.8 \%)$, seguidas de objetos en movimiento (14.4\%). En los de tipo físico, la humedad ocupó el primer lugar (52.2\%), seguida de iluminación deficiente y ruido, ambos con $31.1 \%$ y calor excesivo (25.6\%). Entre los PP de tipo psicosocial, el más frecuente fue trabajo repetitivo $(18.9 \%)$, seguido de estrés laboral $(15.6 \%)$. La posición inadecuada encabeza los procesos potencialmente peligrosos de tipo ergonómico (13.3\%), seguida por movilización de peso (12.2\%). Los biológicos más frecuentes fueron los hongos (32.2\%). En 


\section{Cuadro I \\ Distribución del PERSONAL ESTUdiado SEgún ÁReAS, SEXo Y RANGO DE ANTIGÜEdad en UNA Universidad. Valencia, Venezuela, 1999}

\begin{tabular}{|c|c|c|c|c|c|c|c|c|c|c|c|c|c|c|c|c|}
\hline \multirow[t]{3}{*}{ Area } & \multicolumn{4}{|c|}{ Sexo } & \multicolumn{10}{|c|}{ Rango (años) } & \multirow{2}{*}{\multicolumn{2}{|c|}{ Total }} \\
\hline & \multicolumn{2}{|c|}{ Masculino } & \multicolumn{2}{|c|}{ Femenino } & \multicolumn{2}{|c|}{$0-4$} & \multicolumn{2}{|c|}{$5-9$} & \multicolumn{2}{|c|}{$10-14$} & \multicolumn{2}{|c|}{$15-19$} & \multicolumn{2}{|c|}{$20-25$} & & \\
\hline & $n$ & $\% *$ & $n$ & $\% *$ & $\bar{n}$ & $\% *$ & $\mathrm{n}$ & $\% *$ & $\mathrm{n}$ & $\%^{*}$ & $n$ & $\%^{*}$ & $n$ & $\% *$ & n & $\% / 90$ \\
\hline Depósito de reactivos & 1 & 100 & & & & & & & 1 & 100 & & & & & 1 & 1.11 \\
\hline Bioquímica & 2 & 100 & & & & & & & & & & & 2 & 100 & 2 & 2.22 \\
\hline Tecnología electrónica & 1 & 100 & & & & & & & & & & 100 & & & 1 & 1.11 \\
\hline A picultura & 1 & 100 & & & & & & & & & 1 & 100 & & & 1 & 1.11 \\
\hline Archivo & & & 2 & 100 & & & & & 2 & 100 & & & & & 2 & 2.22 \\
\hline Fitoquímica I & 2 & 40 & 3 & 60 & 1 & 20 & & & 3 & 60 & & & 1 & 20 & 5 & 5.56 \\
\hline N utrición animal ${ }^{\ddagger}$ & - & & - & & & & & & & & & & & & - & - \\
\hline Genética & 1 & 100 & & & & & 1 & 100 & & & & & & & 1 & 1.11 \\
\hline Microbiología general y aplicada & 1 & 50 & 1 & 50 & & & & & & & 1 & 50 & 1 & 50 & 2 & 2.22 \\
\hline Mecánica de fluidos & 1 & 100 & & & & & & & & & & & 1 & 100 & 1 & 1.11 \\
\hline Biología & - & & 1 & 100 & 1 & 100 & & & & & & & & & 1 & 1.11 \\
\hline Almacén & 3 & 100 & & & & & & & & & 1 & 30 & 2 & 70 & 3 & 3.33 \\
\hline Biblioteca & 14 & 48.3 & 15 & 51.7 & 7 & 24 & 5.17 & & & & 6 & 21 & 11 & 38 & 29 & 32.22 \\
\hline Sircas & & & 1 & 100 & & & & & 1 & 100 & & & & & 1 & 1.11 \\
\hline Zoología & 2 & 100 & & & & & & & & & & & 2 & 100 & 2 & 2.22 \\
\hline Fisiología vegetal & 1 & 100 & & & & & & & & & & & 1 & 100 & 1 & 1.11 \\
\hline Carne-leche & 1 & 100 & & & 1 & 100 & & & & & & & & & 1 & 1.11 \\
\hline Entomologías & & & 1 & 100 & & & & & 1 & 100 & & & & & 1 & 1.11 \\
\hline Q uímica I & 2 & 66.6 & 1 & 33.3 & 1 & 33.3 & & & 1 & 33.3 & & & 1 & 33.3 & 3 & 3.33 \\
\hline Bioingeniería & 2 & 100 & & & 2 & 100 & & & & & & & & & 2 & 2.22 \\
\hline Mantenimiento & 14 & 100 & & & 5 & 36 & 3 & 21.5 & 1 & 7 & 4 & 28.5 & 1 & 7 & 14 & 15.55 \\
\hline Autoclave (microbiología) & 1 & 100 & & & & & & & & & & & 1 & 100 & 1 & 1.11 \\
\hline \multicolumn{17}{|l|}{ Anatomía animal $^{\ddagger}$} \\
\hline Computación & 8 & 53.3 & 7 & 46.7 & 1 & 6.6 & 213.5 & 1 & 6.6 & 6 & 0 & 5 & 33.3 & 15 & 16.67 & \\
\hline Total & 58 & & 32 & & 19 & 21.1 & 11 & 12.2 & 11 & 12.2 & 20 & 22.2 & 29 & & 90 & 100.0 \\
\hline
\end{tabular}

SIRC A : Servicio de Investigación-Reactivos, compañía por acciones

* \% sobre el número total de trabajadores por área

₹ Sólo encuesta de área

$\S$ Sin encuesta de área

cada categoría, los restantes tuvieron porcentajes menores a 20. Estos fueron calculados con base en el total de trabajadores, tomando en consideración que un trabajador puede estar expuesto a más de un PP, en una misma categoría y, además, a diversos PP en diversas categorías.

El antecedente médico personal más reconocido fue alergia (30; 33.3\%), seguido de hábito alcohólico $(29 ; 32.2 \%)$ y de problemas dermatológicos $(22 ; 24.4 \%)$. El resto de los síntomas fueron referidos en un porcentaje menor a 20. Sesenta y un trabajadores, refirieron consumir licor. De éstos, 50 (81.97\%) consumen ocasionalmente y $11(18.03 \%)$, lo hace el fin de sema- na. Veintiún trabajadores (18.9\%) fuman. De éstos, 14 $(66.66 \%)$ consumen menos de media cajetilla de cigarrillos/día y siete $(33.33 \%)$, más de media cajetilla diaria.

Con referencia al perfil patológico, los signos y síntomas presentados por los trabajadores durante los últimos seis meses, se expresan en el cuadro III. Se agruparon en 12 las principales categorías, y las restantes como "otros". El 81.1\% (73), refirieron síntomas.

En el cuadro IV se describe el número de trabajadores que reportaron signos y síntomas, de acuerdo con el sexo y el área de trabajo. 


\section{Cuadro II \\ Grado de Riesgo, Según La INTENSIDAd DEL RIESGO Y LAS CARACTERÍSTICAS DE EXPOSICIÓN, DEL PERSONAL ESTUDIADO EN UNA UNIVERSIDAD. Valencia, Venezuela, 1999}

\begin{tabular}{lccc} 
Area & $\begin{array}{c}\text { Características } \\
\text { de exposición }\end{array}$ & $\begin{array}{c}\text { Intensidad } \\
\text { de riesgo }\end{array}$ & $\begin{array}{c}\text { Grado } \\
\text { de riesgo }\end{array}$ \\
Depósito de reactivos & 2 & 3 & 3 \\
\hline Bioquímica & 1 & 2 & 2 \\
\hline Tecnología electrónica & 2 & 1 & 2 \\
\hline A picultura & 1 & 1 & 1 \\
\hline Archivo & 2 & 2 & 2 \\
\hline Fitoquímica I & 2 & 3 & 3 \\
\hline Nutrición animal & 1 & 3 & 2 \\
\hline Genética & 2 & 2 & 2 \\
\hline Microbiología general y aplicada & 3 & 3 & 3 \\
\hline Mecánica de fluidos & 3 & 3 & 3 \\
\hline Biología & 3 & 3 & 3 \\
\hline Almacén & 3 & 3 & 3 \\
\hline Biblioteca & 2 & 2 & 2 \\
\hline Servicio de investigación-reactivos & 1 & 1 & 1 \\
\hline Zoología & 2 & 2 & 2 \\
\hline Fisiología vegetal & 1 & 1 & 1 \\
\hline Carne-leche & 2 & 3 & 3 \\
\hline Entomología & 2 & 2 & 2 \\
\hline Química I & 2 & 2 & 2 \\
\hline Bioingeniería & 1 & 1 & 1 \\
\hline Mantenimiento & 3 & 3 & 3 \\
\hline Autoclave (microbiología) & 2 & 2 & 2 \\
\hline Anatomía animal & 3 & 3 & 3 \\
\hline Computación & 2 & 1 & 2 \\
\hline
\end{tabular}

En relación con la utilización de EPP por los trabajadores de estas áreas, en la biblioteca, donde refieren estar expuestos a hongos presentes en el ambiente, sólo $34.5 \%$ informó del uso de mascarilla. En mantenimiento el uso de EPP se da de la siguiente manera: protector de oídos, $7.4 \%$; overall, $42.8 \%$; botas, $71.4 \%$; guantes, $57.1 \%$, mascarilla, $42.9 \% \mathrm{y}$, a pesar de la presencia de ruido, sólo una persona $(7.4 \%)$, usa protectores auditivos. Las otras dos dependencias con GR=3 que usaron alguno, fueron depósito de reactivos (su único trabajador usaba overall, botas y mascarilla) y en microbiología general y aplicada, 50\% usaba guantes.

El estudio estadístico de los datos permitió establecer una correlación positiva significativa entre la antigüedad laboral de los trabajadores de la UN y el número de signos y síntomas referidos por los mismos $(r=0.339, p<0.01)$. En cuatro dependencias, más de $50 \%$ de la población femenina presentó signos y síntomas.

No se encontró una correlación significativa entre el número de signos y síntomas de acuerdo con el sexo

\section{Cuadro III \\ Distribución de los tRaBajadores SEGÚN LOS PRINCIPALES SIGNOS Y SINTTOMAS PRESENTADOS DURANTE LOS ÚLTIMOS SEIS MESES en una universidad.VAlencia, Venezuela, 1999}

\begin{tabular}{lcc} 
Signos y síntomas & $n$ & $\% *$ \\
Cefalea & 27 & 30 \\
\hline Alergias & 18 & 20 \\
\hline Prurito & 13 & 14.4 \\
\hline Irritación ocular & 13 & 14.4 \\
\hline Cólicos & 13 & 14.4 \\
\hline Fatiga & 12 & 13.3 \\
\hline Ansiedad & 11 & 12.2 \\
\hline Mareos & 10 & 11.1 \\
\hline Debilidad & 9 & 10 \\
\hline Presión arterial alta & 8 & 8.9 \\
\hline Falla visual & 8 & 8.9 \\
\hline Tos & 8 & 8.9 \\
\hline Otros & 70 & 77.8
\end{tabular}

* Con base en el total de trabajadores estudiados (90), tomando en consideración que un trabajador puede referir más de un signo o síntoma

\section{Cuadro IV \\ Distribución de LA POBLACIÓN ESTUdiada DE ACUERDO CON SIGNOS Y SINTOMAS REPORTADOS, EN LOS ÚlTIMOS SEIS MESES, SEGÚN SEXO Y ÁREA, en UNA UNiversidad. Valencia, Venezuela, 1999}

\begin{tabular}{|c|c|c|c|c|}
\hline \multirow{3}{*}{ Area } & \multicolumn{4}{|c|}{ No. de trabajadores con signos o síntomas } \\
\hline & \multirow{2}{*}{$\frac{\text { Masculino }}{n \text { \%/total* }}$} & \multirow{2}{*}{$\frac{\text { Femenino }}{n \text { \% } / \text { total }\left.\right|^{*}}$} & \multicolumn{2}{|c|}{ Total } \\
\hline & & & $\bar{n}$ & $\% *$ \\
\hline Depósito de reactivos & 1100 & & 1 & 100 \\
\hline Bioquímica & 150 & & 1 & 50 \\
\hline Tecnología electrónica & 1100 & & 1 & 100 \\
\hline \multicolumn{5}{|l|}{ Apicultura } \\
\hline Archivo & & 2100 & 2 & 100 \\
\hline Fitoquímica I & 120 & 360 & 4 & 100 \\
\hline Genética & 1100 & 1 & 100 & \\
\hline Microbiología general y aplicada & 150 & 150 & 2 & 100 \\
\hline Mecánica fluidos & 1100 & & 1 & 100 \\
\hline Biología & & 1100 & 1 & 100 \\
\hline Almacén & 3100 & & 3 & 100 \\
\hline Biblioteca & $10 \quad 34$ & 1345 & 23 & 79 \\
\hline $\begin{array}{l}\text { Servicio de investigación- } \\
\text { reactivos }\end{array}$ & & & 0 & 0 \\
\hline Zoología & 2100 & & 2 & 100 \\
\hline Fisiología vegetal & 1100 & & 1 & 100 \\
\hline \multicolumn{5}{|l|}{ Carne-leche } \\
\hline Entomología & 1100 & & 1 & 100 \\
\hline Química I & 267 & 133 & 3 & 100 \\
\hline Bioingeniería & 2100 & & 2 & 100 \\
\hline Mantenimiento & 1071.4 & & 10 & 71.4 \\
\hline Autoclave (microbiología) & 1100 & & 1 & 100 \\
\hline Anatomía animal & - & - & - & \\
\hline Computación & 533 & $8 \quad 53$ & 13 & 86.6 \\
\hline
\end{tabular}


$(r=-0.21)$ ni de acuerdo con la edad de la población estudiada $(r=0.28)$.

En relación con los PP específicos por área de trabajo los resultados se indican en el cuadro $\mathrm{V}$, y es mantenimiento la que presenta el mayor número de PP (44), seguida de bioquímica (20), depósito de reactivos y microbiología general y aplicada, con 15 PP en cada una.

\section{Discusión}

Los datos obtenidos permitieron analizar las condiciones de exposición laboral en la que se desempeñan los trabajadores de la universidad en estudio. Los resultados indican la inexistencia de PSSO, requisito indispensable para el mantenimiento de la calidad de vida de sus trabajadores, al prevenir, tanto efectos potencialmente adversos a la salud, como ocurrencia de accidentes.
En la revisión bibliográfica no se identificaron trabajos similares que pudiesen ser comparables. Hornsby P y colaboradores, ${ }^{2}$ quienes hicieron un trabajo en la Universidad de Virginia, orientaron su estudio hacia el establecimiento de un programa clínico, de promoción de la salud y prevención de enfermedades. El trabajo de Emery R y colaboradores, ${ }^{1}$ compara los programas de salud y seguridad entre instituciones de minorías y no-minorías de los Estados Unidos de América. La similitud que tiene con el presente estudio es que sólo identificaron los PP químicos, físicos y biológicos, pero no midieron los niveles relativos de cada uno de ellos.

En el cuadro I se puede observar que existen seis áreas $(23 \%)$, donde hay un porcentaje igual o mayor a $50 \%$ de mujeres sobre hombres, y en archivo, Servicio de investigación-reactivos SIRCA, entomología y biología, sólo laboran mujeres. Esto es importante por su particular vulnerabilidad en periodos de embarazo y

\section{Cuadro V \\ Distribución de los procesos peligrosos según Áreas laborales estudiadas en UNA UNiversidad. Valencia,Venezuela, 1999}

\begin{tabular}{|c|c|c|c|c|c|c|c|c|c|c|c|c|}
\hline \multirow[b]{3}{*}{ Area } & \multirow[b]{3}{*}{ Código } & \multicolumn{10}{|c|}{ Tipos de procesos peligrosos presentes } & \multirow[b]{3}{*}{ Total } \\
\hline & & \multicolumn{2}{|c|}{ Químico } & \multicolumn{2}{|c|}{ Mecánico } & \multicolumn{2}{|c|}{ Físico } & \multicolumn{2}{|c|}{ Psicosocial } & \multicolumn{2}{|c|}{ Ergonómico } & \\
\hline & & $\bar{n}$ & $\% *$ & n & $\%^{*}$ & $\bar{n}$ & $\% *$ & $n$ & \%* & $\mathrm{n}$ & \%* & \\
\hline Depósito de reactivos & 1 & 7 & 47 & 0 & 0 & 6 & 40 & 1 & 6.5 & 1 & 6.5 & 15 \\
\hline Bioquímica & 2 & 9 & 45 & 1 & 5 & 8 & 40 & 2 & 10 & 0 & 0 & 20 \\
\hline Tecnología electrónica & 3 & 4 & 40 & 5 & 50 & 1 & 10 & 0 & 0 & 0 & 0 & 10 \\
\hline A picultura & 4 & 1 & 33 & 2 & 67 & 0 & 0 & 0 & 0 & 0 & 0 & 3 \\
\hline Archivo & 5 & 0 & 0 & 0 & 0 & 4 & 50 & 2 & 25 & 2 & 25 & 8 \\
\hline Fitoquímica I & 6 & 9 & 100 & 0 & 0 & 0 & 0 & 0 & 0 & 0 & 0 & 9 \\
\hline $\bar{N}$ utrición animal ${ }^{\ddagger}$ & 7 & & & & & & & & & & & \\
\hline Genética & 8 & 2 & 67 & 0 & 0 & 0 & 0 & 1 & 33 & 0 & 0 & 3 \\
\hline Microbiología general y aplicada & 9 & 5 & 33 & 2 & 13 & 7 & 47 & 0 & 0 & 1 & 7 & 15 \\
\hline Mecánica fluidos & 10 & 5 & 50 & 3 & 30 & 2 & 20 & 0 & 0 & 0 & 0 & 10 \\
\hline Biología & 11 & 3 & 60 & 0 & 0 & 2 & 40 & 0 & 0 & 0 & 0 & 5 \\
\hline Almacén & 12 & 4 & 57 & 0 & 0 & 2 & 29 & 1 & 14 & 0 & 0 & 7 \\
\hline Biblioteca & 13 & 4 & 29 & 0 & 0 & 5 & 36 & 3 & 21 & 2 & 14 & 14 \\
\hline Servicios de Investigación-reactivos ${ }^{\S}$ & 14 & 5 & 100 & 0 & 0 & 0 & 0 & 0 & 0 & 0 & 0 & 5 \\
\hline Zoología & 15 & 6 & 86 & 0 & 0 & 1 & 14 & 0 & 0 & 0 & 0 & 7 \\
\hline Fisiología vegetal & 16 & 5 & 83 & 0 & 0 & 1 & 17 & 0 & 0 & 0 & 0 & 6 \\
\hline Carne-leche & 17 & 0 & 0 & 4 & 67 & 2 & 33 & 0 & 0 & 0 & 0 & 6 \\
\hline Entomologías & 18 & 1 & 33.3 & 0 & 0 & 1 & 33.3 & 0 & 0 & 1 & 33.3 & 3 \\
\hline Q uímica I & 20 & 7 & 64 & 0 & 0 & 4 & 36 & 0 & 0 & 0 & 0 & 11 \\
\hline Bioingeniería & 22 & 0 & 0 & 1 & 20 & 2 & 40 & 1 & 20 & 1 & 20 & 5 \\
\hline Mantenimiento & 23 & 15 & 34 & 9 & 20 & 10 & 23 & 6 & 14 & 4 & 9 & 44 \\
\hline Autoclave (microbiología) & 24 & 1 & 33 & 0 & 0 & 2 & 67 & 0 & 0 & 0 & 0 & 3 \\
\hline$\overline{\text { Anatomía animal }^{\ddagger}}$ & 25 & & & & & & & & & & & \\
\hline Computación & 26 & 1 & 5 & 0 & 0 & 8 & 47 & 4 & 24 & 4 & 24 & 17 \\
\hline $\begin{array}{l}\text { * \% sobre el número total de trabaja } \\
\text { ₹ Sólo encuesta de área } \\
\text { § Sin encuesta de área }\end{array}$ & r área & & & & & & & & & & & \\
\hline
\end{tabular}


lactancia. ${ }^{*}$ Los antecedentes personales referidos podrían exacerbar los potenciales efectos adversos relacionados con la exposición de tipo ocupacional y hacer a los empleados más vulnerables por su estado basal de salud.

El consumo de alcohol de $67.8 \%$ de los trabajadores, aunque no se considere alcoholismo crónico, es relevante ya que el etanol, aun en pequeñas dosis en el organismo, interactúa con medicamentos, agentes tóxicos ocupacionales, inductores enzimáticos, etcétera. Esta interacción en muchos casos potencia los efectos adversos de solventes orgánicos (xileno, tolueno, tricloroetileno), presentes en varias de las áreas evaluadas en este estudio. ${ }^{8}$ Igualmente altera el estado de alerta y el tiempo de reacción, lo cual podría favorecer la accidentabilidad.

En los laboratorios, donde es más común el uso de reactivos, el mayor porcentaje de PP de tipo químico, correspondió a polvos, seguido de solventes, ácidos y bases fuertes, que se utiliza a menudo sin que se tomen las medidas de precaución necesarias. Con relación al PP físico más frecuente (humedad), ésta reviste especial importancia en ambientes como la biblioteca. Condiciones favorables de temperatura y humedad allí existentes, podrían exacerbar la proliferación de hongos en los libros. Esto, aunado a la presencia de polvos, puede generar patologías de tipo respiratorio y cutáneo, las cuales podrían prevenirse con modificación de las condiciones ambientales (ventilación, humidificación). Los problemas generados por el ruido (referido por $31.1 \%$ de los encuestados) suelen pasar desapercibidos por tratarse de algo cotidiano. Sin embargo, es un factor que afecta la labor educativa ya que incrementa la fatiga nerviosa y la agresividad, dando lugar a problemas de comportamiento y a conflictos; disminuye el nivel de atención y obliga al profesorado a forzar la voz para poder mantener la comunicación, dando lugar a la aparición de disfonías y estrés.*

En los PP psicosociales el trabajo repetitivo fue el más reportado, seguido por el estrés laboral, lo cual podría influenciar la parte anímica y producir desmotivación para el trabajo y baja autoestima cuando no se refuerzan las habilidades propias del trabajador. Una variante específica del estrés en el ámbito docente es el llamado síndrome de Burn-out o síndrome de quemado, caracterizado por la aparición de sentimientos de cansancio emocional y fatiga, actitudes de despersonalización hacia el alumnado y una pérdida de la realización personal en el trabajo., ${ }^{*, 10}$ Los PP ergo-

\footnotetext{
* Sindicato de trabajadores de la enseñanza de Euzkadi (STEE-EILAS). Riesgos laborales en el sector de la enseñanza. Disponible en: http:/ / www.stee-eilas.com [Consultado el 24/09/2001]
}

nómicos, junto con los de tipo psicosocial, podrían agravar situaciones prexistentes o inducir a la aparición de síntomas vinculados al trabajo. En los PP biológicos, aunque las enfermedades infecciosas están bastante controladas en la población general, el personal de la biblioteca y los que trabajan con animales están expuestos a una variedad de agentes biológicos que causan infección y enfermedades que son, a veces, difíciles de controlar. La hepatitis, la tuberculosis y la shigellosis (disentería), son riesgos profesionales para trabajadores de los laboratorios. ${ }^{11}$

Se pudo observar que, en general, las patologías referidas por los trabajadores entrevistados (cuadro III) no son específicas de exposición a un riesgo en particular y por lo tanto, difíciles de cuantificar. Si además tomamos en cuenta que en la mayoría de las áreas coexisten diversos tipos de riesgos que pudieran producir una afectación específica, no es menos cierto que los antecedentes personales o familiares existentes en los trabajadores, aunados al tiempo de permanencia en dichas áreas y a la conocida exposición extralaboral, podrían contribuir a la aparición de dichos síntomas. Es difícil atribuirlos a un agente tóxico específico sin la utilización de biomarcadores objetivos de exposición y de efecto, que permitan establecer una relación causa-efecto consistente entre estas patologías y la exposición laboral. ${ }^{12}$ Síntomas neurológicos como cefalea $(27 \%)$, fatiga $(12 \%)$ y ansiedad $(11 \%)$, pueden ser sutiles, no específicos y difíciles de detectar aun en un examen neurológico común y podrían asociarse, tanto a intensa-extensa carga de trabajo, como a tensión o estrés laboral. Representan, además, síntomas tempranos de neurotoxicidad y pueden ocurrir a bajos niveles de exposición, pasando de esta forma inadvertidos, y para los cuales no se toman medidas de control necesarias para evitar efectos adversos mayores.

La correlación positiva significativa, determinada entre la antigüedad de los trabajadores de la universidad estudiada y los síntomas referidos por los mismos, nos podrían sugerir una relación que habla a favor de que en la medida en que los trabajadores tienen mayor tiempo laborando en su área de trabajo, presentarían una serie de signos y síntomas relacionados con los riesgos a los cuales están expuestos constantemente. Sería muy apresurado aceptar, de entrada, esta afirmación. No obstante, si a esto sumamos el estado de fatiga del personal y el régimen de remuneraciones deprimidas de la UN, podrían implicar un deterioro de la calidad de vida.

Podemos observar que el GR no necesariamente es directamente proporcional al número de trabajadores. El cuadro II muestra que existen diversas áreas que presentan riesgo elevado, tal es el caso del depósito de reactivos, donde labora sólo un trabajador y está 
catalogada como un $G R=3$, ya que almacena una serie de sustancias tóxicas, presenta condiciones inseguras (falta de clasificación y ordenamiento de las sustancias dentro del sitio), y no se utiliza el equipo de protección personal específico de forma habitual. La respuesta obtenida acerca del conocimiento que tienen sobre los procesos potencialmente peligrosos a los que están expuestos llama la atención, pues no existe en la universidad un programa de seguridad y salud ocupacional, ni siquiera un Comité de Higiene y Seguridad, como es obligatorio según LOPCYMAT, ${ }^{4}$ que tuviera la responsabilidad del entrenamiento de los trabajadores en esta materia. En el cuadro IV se observa que en 15 áreas, cuyo número de trabajadores es $\leq 3$, todos refirieron signos o síntomas. Por ser esta población tan pequeña sería muy difícil emitir un juicio objetivo en torno a las patologías presentadas. Sin embargo, observamos que las áreas con el personal más numeroso, tienen un elevado porcentaje de individuos sintomáticos (biblioteca 79, computación, 86.6 y mantenimiento, 71.4), aunque la única que corresponde a la máxima calificación de GR es mantenimiento. Esto era de esperarse ya que en dicha área están presentes 44 de los PP estudiados (cuadro V), incluyendo variadas condiciones inseguras que conducen a un mayor grado de accidentabilidad.

Entre las limitaciones del presente estudio, podríamos mencionar: a) debido a la particularidad de la población estudiada, los resultados descritos no reflejan las condiciones presentes en otro tipo de instituciones académicas de nuestro país, por ejemplo, las privadas (no gubernamentales); b) el grado de PP sólo indicó presencia o ausencia, y c) es posible que se incluyan sesgos en las respuestas de los individuos encuestados por la debilidad de su confiabilidad. Estas limitaciones representan una buena oportunidad para el diseño y refinamiento de estudios similares en nuestras universidades.

\section{Conclusiones}

- En la universidad estudiada se presentan variados procesos potencialmente peligrosos en las diferentes áreas laborales por las características inherentes de su misión.

- Las patologías referidas son inespecíficas y por lo tanto difíciles de asociar con un proceso en particular.

- Hasta la fecha del estudio no existía un Programa de Seguridad y Salud Ocupacional y no se llevan registros de ausentismo, accidentabilidad, morbilidad y mortalidad, que pudieran asociarse con la exposición ocupacional. Por lo tanto, se recomienda:

- La creación de un Servicio de Salud Ocupacional e Higiene Industrial, con carácter multidisciplinario, que dé prioridad a las actividades preventivas y donde se lleven adecuados registros sobre siniestrabilidad laboral, morbilidad y mortalidad, que permitan la detección temprana de enfermedades $\mathrm{y}$ accidentes ocupacionales.

- Cumplir con los requisitos mínimos señalados en la legislación venezolana (LOPCYMAT), en materia de prevención de riesgos laborales.

- El diseño de programas de vigilancia periódica con la intención de prevenir, identificar, evaluar y controlar los diferentes riesgos presentes en cada una de las áreas laborales de la universidad, con el fin de evitar el efecto negativo sobre la salud de los trabajadores, deberá incluir examen preempleo periódico, monitoreo ambiental y biológico, estudios ergonómicos de los puestos de trabajo y cumplimiento de las normas establecidas de higiene y seguridad laboral.

- Establecer un programa educativo sobre los potenciales efectos adversos del ambiente laboral, así como las medidas de prevención y de atención de urgencias, con el objetivo de mantener un ambiente de trabajo saludable donde ellos mismos sean garantes de su propio bienestar biopsicosocial.

\section{Referencias}

1. Emery R, D eclos G, Cooper S, Hardi R. Evaluating the relative status of health and safety programs for minority academic and research institutions. Am Ind Hyg Assoc J 1998;59:882-888.

2. Hornsby P, Reeve R, G waltney J, Parson B, Morse RM.The University of Virginia Health Promotion and Disease Prevention Program. Am J Prev Med 1997;13(1):36-44.

3. Christman E, Gandsman E. Radiation safety as part of a comprehensive university occupational health and safety program. Health Phys Soc 1994;66(5):581-584.

4. LO PCYMAT. Ley O rgánica de Prevención, Condiciones y Medio Ambiente de Trabajo. Congreso de la República de Venezuela. Gaceta official N o. 3850, 18-7-1986.

5. 0 maña E. Sistema de vigilancia de las condiciones de trabajo y de la salud de los trabajadores. Impacto de los cambios normativos y políticos en Venezuela. Simposio de la Red de Estudios del Trabajo; 1999 Julio 2630; San Antonio de los Altos, Venezuela. 
6. Martínez C, O maña E. Sistemas de información en salud ocupacional. Reunión Andina en Salud de los Trabajadores. La Paz, Bolivia, 1994:67-71. 7. $\mathrm{N}$ iland J. Industrial Hygiene. En: $\mathrm{C}$ arl Zenz, ed. $\mathrm{O}$ ccupational Medicine. Third Edition. St. Louis (MO ): Editorial Mosby, 1994:1012-1060.

8. Barret CJ,Vainio H, Peakall D, G oldstein B. $12^{\text {th }}$ Meeting of the scientific group on methodologies for the safety evaluation of chemicals: Susceptibility to environmental hazards. Environ Health Perspect 1997;105(4): 699-737.

9. Betancourt 0. Para la enseñanza e investigación de la salud y seguridad en el trabajo. Q uito:FUN SAD-O PS/O MS, 1999.
10. Alfonzo L. Condiciones y medio ambiente de trabajo en las unidades de información universitarias: caso UCV (tesis).C aracas: Universidad C entral de Venezuela, 1994.

11. Tennassee M. Condiciones de salud de los trabajadores del area andina. Reunión Andina en Salud de los Trabajadores. La Paz, Bolivia, 1994: 25-39.

12.Axelson 0 , Hogstedt CH.The health effects of solvents. En: $\mathrm{C}$ arl Zenz, ed. 0 ccupational Medicine.Third Edition. St. Louis (MO): Editorial Mosby, 1994:764-778. 